\title{
CIENTÍFICOS ESPAÑOLES EN EL REINO UNIDO (1750-1830*
}

\author{
Manuel Valera \\ U. D. Historia de la Medicina, Universidad de Murcia (España) \\ Juan Francisco López Sánchez \\ Departamento de Física, Escuela Politécnica de Cartagena, Universidad de Murcia (España)
}

\section{Carlos López Fernández}

I. N. B. Alfonso X, Murcia (España)

\section{RESUMEN}

El objeto del presente trabajo es dar cuenta detallada del flujo casi continuo de españoles que, por uno u otro motivo, realizaron alguna estancia en el Reino Unido desde mediados del siglo XVIII hasta los años treinta del siglo XIX. Exponemos primeramente los rasgos más sobresalientes de la actividad desplegada por los científicos españoles en Inglaterra, encuadrada en sus respectivas áreas de trabajo, y posteriormente damos noticia de los españoles asociados a la institución científica británica más emblemática de la época, la Royal Society of London.

\section{SUMMARY}

The main object of this article is to explain in detail the continous movement of Spanish people, that by some reason, lived in the U. K. from mid XVIII'th century until the 30 's in the XIX'th century. Hereby is exposed the main features carried out by Spanish scientist in England, as well as their respective work areas. Afterwords, are numbered the Spanish people associated to the most emblematic British science institution of those days, the Royal Society of London.

* Este artículo forma parte del Proyecto "Relaciones científicas entre España y Gran Bretaña..." (DGICYT PS92-0129). Uno de nosotros (MV) se ha beneficiado también de una estancia financiada por la DGICYT en el Wellcome Institute for the History of Medicine of London. Una versión más reducida del trabajo se presentó en el Congreso Internacional de Historia de la Ciencia celebrado en agosto de 1993 en Zaragoza. 
MANUEL VALERA, JUAN FRANCISCO LÓPEZ SÁNCHEZ Y CARLOS LÓPEZ FERNÁNDEZ

\section{EL IMPULSO DE LA ESPAÑA ILUSTRADA A LAS RELACIONES CIENTÍFICAS CON GRAN BRETAÑA}

Debido al peculiar desarrollo de la ciencia española, las relaciones científicas entre España y otros países gozan de especial relevancia, constituyendo su estudio una materia de gran interés para el conocimiento de nuestro pasado científico.

La situación geográfica de nuestro país ha constituido, sin duda, un factor determinante en las relaciones exteriores españolas, pues al ser Francia y Portugal los únicos países europeos con los que España tiene frontera terrestre, la mayor parte de la cultura literaria o científica, así como los adelantos de la técnica, de la economía o de la política han llegado por ese camino, filtrados generalmente por el pensamiento francés ${ }^{1}$. Esta situación geográfica española era contemplada negativamente por Humboldt, pues, en sus propias palabras, al estar España sólo unida con Francia y separada del resto de Europa, "en todas partes aparece la desventaja del influjo unilateral de la literatura francesa"2.

La llegada al trono español de la Casa de Borbón no hizo sino acentuar este predominio de la influencia francesa, llegándose, en opinión de Mendoza y Ríos, al extremo de desconocer lo que se publicaba en otros países, pues "todo el comercio de libros estrangeros que hacen los mercaderes en España se reduce casi enteramente a los que salen a luz en Francia y (...) en quanto a la Inglaterra, Italia y Alemania donde se escribe mucho y bueno, carecemos hasta de la noticia de las impresiones que se executan"3.

En cualquier caso, bien sea por razones de proximidad geográfica o de intereses políticos o dinásticos, está claro que el país con el que España habitualmente ha mantenido una relación científica de privilegio ha sido Francia. Son mucho menos conocidas, sin embargo, las relaciones entre España y Gran Bretaña, pues entre estos países no ha habido siempre una fluida relación ${ }^{4}$, al menos en el ámbito científico.

1 GARMA (1978), "Producción matemática y cambios en el sistema productivo en la España de finales del siglo XVIII". En Homenaje a Julio Caro Baroja, Madrid. 431-447; pp. 432-433.

2 Carta de Humboldt a David Frienlander de 16 de diciembre de 1796, reproducida en "Juicios de Wilhem Von Humboldt sobre el carácter español y los ilustrados del 1800". Traducción y notación por Justo Gárate. Asclepio, 17, 273-278, 1965.

3 Museo Naval, ms. 2317.

4 Por no hablar de franco enfrentamiento. Para limitarnos solamente al siglo XVIII, el número de conflictos en que se vieron envueltos ambos países fue extraordinariamente elevado, siempre con un trasfondo de intereses económicos en pugna. Recordemos someramente la secuencia cronológica de los conflictos bélicos que enfrentaron a España y Gran Bretaña desde finales del siglo XVII hasta el fin de la época napoleónica: 1) 1700-1713, Guerra de Sucesión a la Corona de España, concluida con la paz de Utrecht; 2) 1718, escaramuza originada por la ocupación de Cerdeña y demostración de fuerza de la escuadra inglesa en el Cabo Passaro; 3) 1738-1748, nueva guerra entre España y Gran Bretaña, complicada a partir de 1742 por la participación de ambos países en bandos opuestos en la Guerra de Sucesión a la Corona de Austria, hasta la paz de Aquisgrán de 1748; 4) 1762-1763, participación de España en la 
Ahora bien, el hecho de no ser muy conocidas no quiere decir que no hayan existido, especialmente en la segunda mitad del siglo XVIII.

Con el proyecto ilustrado de Carlos III se pusieron en marcha una serie de mecanismos encaminados a superar el atraso científico español. En este contexto se crearon numerosas instituciones científico-técnicas (Real Gabinete de Máquinas, Real Gabinete de Historia Natural, Laboratorio Metalúrgico, Laboratorio de Química Aplicada a las Artes, etc.), fueron contratados reputados científicos extranjeros (Proust, Chabaneau, Loeffling, Godin, Vimercati, Giannini, Bowles, Herrgen, etc.), y gran cantidad de pensionados españoles fueron enviados hacia diversos países europeos, especialmente hacia Francia. Pero aunque en mucho menor número que el correspondiente al país vecino, también hubo una cantidad relativamente importante de científicos españoles que marcharon al Reino Unido, sobre todo durante el último cuarto de siglo: C. Gómez Ortega, A. y C. Gimbernat, J. M. Ruiz de Luzuriaga, J. Mendoza y Ríos, A. de Betancourt, C. Sánchez, C. y E. Boutelou, etc.

Con el cambio de siglo, las relaciones científicas hispano-británicas adquirieron un fuerte matiz político, pues la mayor parte de los científicos españoles que viajaron o se establecieron en Gran Bretaña durante el primer tercio del siglo XIX, lo hicieron por razones de índole política. De hecho, el momento culminante del flujo de científicos españoles al Reino Unido, se produjo tras el aplastamiento de Riego y la restauración del régimen absolutista en 1823. En esos años veinte del pasado siglo hubo una auténtica oleada de exilados españoles en Londres, puesto que Francia ya no era, como en otras ocasiones, el refugio natural de los mismos.

Dada la conformación social de los grupos liberales españoles, constituidos en gran medida por capas medias y sectores profesionales, éstos fueron precisamente los grupos sociales predominantes en el exilio de 1823: militares, abogados, sacerdotes, comerciantes, literatos y médicos 5 . Todos ellos eran profesionales formados en su mayor parte en la última época de la Ilustración y, como el propio hecho de su exilio pone de manifiesto, imbuidos de un liberal "espíritu ilustrado", por lo que pueden ser considerados como los últimos representantes de la España científica ilustrada en el Reino Unido, con cuyos principios políticos liberales casi todos ellos se sentían

Guerra de los Siete Años, que desde 1756 enfrentaba a Francia e Inglaterra, y que terminaría con la firma de la paz de París; 5) 1779-1783, participación española en la Guerra de la Independencia americana, finalizada con la paz de Versalles; 6) 1796-1802, participación de nuevo junto a Francia, finalizada con la paz de Amiens; 7) por último, 1804-1809, cierra este ciclo de guerras casi permanentes entre España y Gran Bretaña, que caracterizó todo el siglo XVIII.

Poco después, la participación de Inglaterra junto a España en la lucha contra Napoleón, invirtió la tendencia imperante durante el siglo anterior, y abrió un período de colaboración que llevaría, finalmente, a la firma de un Tratado de paz y amistad en 1814 que ya no se rompería en todo el siglo XIX. (NADAL, J. (1978): Comercio exterior y subdesarrollo. España y Gran Bretaña de 1772 a 1914, Madrid, pp. 114-115).

5 LLORENS, V. (1968): Liberales y románticos. Una emigración española en Inglaterra. 1823-1834, 2. ${ }^{a}$ ed., Madrid, p. 24. 
identificados. Entre los científicos más destacados de este exilio podemos señalar, entre otros, a J. M. de Aréjula, M. Lagasca, F. Bauzá, M. Seoane, etc.

Sobre este flujo casi continuo de españoles que, por uno u otro motivo, realizaron alguna estancia en el Reino Unido desde mediados del siglo XVIII hasta los años treinta del siglo XIX, versa el presente trabajo. Hablaremos en primer lugar de la actividad allí realizada, encuadrándola en su correspondiente área científica, y posteriormente daremos cuenta de los españoles asociados a la institución científica británica más emblemática de la época, la Royal Society of London.

PRINCIPALES ÁREAS DE ACTIVIDAD DE LOS CIENTÍFICOS ESPAÑOLES EN EL REINO UNIDO

\section{Astronomía y Náutica}

Entre los diferentes ámbitos científicos donde las relaciones entre España y Gran Bretaña adquirieron especial relevancia, debemos destacar ante todo la Náutica y disciplinas afines: astronomía, cosmografía, hidrografía, cartografía, así como el de los instrumentos científicos con ellas relacionados.

Los primeros científicos españoles pertenecientes a este ámbito que tuvieron alguna relación con el Reino Unido fueron Antonio de Ulloa y Jorge Juan, bien es cierto que en circunstancias muy diferentes: el primero como prisionero de guerra y el segundo en una misión especial.

Al finalizar su misión en el Virreinato del Perú, Antonio de Ulloa partió en octubre de 1744 del puerto de El Callao a bordo del navío francés la Deliverance. Sin embargo, el barco fue apresado por la Armada Británica en Louisbourg, y Ulloa, como prisionero de guerra, fue conducido a Inglaterra, y sus documentos fueron requisados ${ }^{6}$. El 22 de diciembre de 1745 llegaba a Porstmouth en el Sunderland, el mismo navío que lo había apresado.

\footnotetext{
6 Los documentos de Ulloa, supuestamente referidos a la gobernación de Indias y portadores de información del mayor interés logístico, fueron igualmente trasladados a Inglaterra. Pero al parecer, Ulloa había arrojado al mar todos los papeles que pudieran comprometer la seguridad de las colonias o la imagen internacional de España, por lo que la documentación requisada sólo contenía el diario de las observaciones científicas.

Una información muy completa de la estancia de Ulloa en Londres se encuentra en WHITAKER, A. (1935) "Antonio de Ulloa", The Hispanic American Historical Review, 15, 155-194; y sobre todo, en otro trabajo del mismo autor: WHITAKER, A. (1966): "Antonio de Ulloa, the Délivrance, and the Royal Society", The Hispanic American Historial Review, 46, 357-370. También el propio Ulloa da cumplida cuenta de su estancia londinense en ULLOA, A.; JUAN, J. (1748): Relación histórica del viage a la America meridional..., Madrid, Imp. Antonio Marín, 5 tomos. (Reimpresión en 3 tomos, con una introducción
} 
Afortunadamente para Ulloa, durante los seis meses que se prolongó su estancia en Inglaterra recibió un trato extremadamente cordial ${ }^{7}$. Entró allí en contacto con el presidente de la Royal Society, Martin Folkes, quien no sólo le facilitó la recuperación de los documentos requisados, sino que le invitó a asistir a varias reuniones de la Sociedad, presentándole siempre como un "eminente extranjero", y además le propuso como miembro de la misma ${ }^{8}$. De este modo, un suceso aparentemente tan grave en un principio, acabó teniendo consecuencias muy favorables para Ulloa, pues su nombramiento como fellow de la Royal Society venía a ser como el reconocimiento internacional de su labor en la medida del grado en el Ecuador.

Una vez le fueron reintegrados sus documentos, Ulloa abandonó Londres, llegando a Madrid en julio de 1746, once años y dos meses después de su partida al Virreinato del Perú.

En octubre de 1748, poco después de la publicación de la Relación histórica.... recibía Jorge Juan una Instrucción reservada del marqués de la Ensenada para trasladarse a Londres, acompañado de dos guardias marinas9. En la Instrucción se especi-

de José P. Merino y Miguel M. Rodríguez, Madrid, 1978). Para la estancia de Ulloa en Inglaterra, véase Tomo II, pp. 535-544.

7 En realidad, la generosidad hacia el enemigo vencido no era un fenómeno infrecuente en la Europa de aquella época, al menos cuando el enemigo era un oficial y un caballero, como lo era Ulloa. Además, en este caso se trataba de un científico que acababa de participar en una importante misión en un remoto lugar, lo cual constituía suficiente garantía en un país como Inglaterra, que se ufanaba de la protección y el fomento de las ciencias.

8 Ulloa asistió a las reuniones de la Royal Society de 17 y 24 de abril y 1 de mayo de 1746 (Archivo de la RS: Journal Book Copy (JBC), 19, pp. 81, 87 y 95). Fue propuesto como Fellow de la Sociedad por Folkes, Lord Stanhope y Cromwell Mortimer $(J B C, 19$, p. 104) y su candidatura, como era preceptivo, fue sometida a votación y aprobada seis meses más tarde (JBC, 19, p. 167).

En todas estas atenciones, aparte de la cortesía natural de Folkes, también debió influir la circunstancia de haber participado Ulloa en la expedición para la medida del grado en el Ecuador. Aunque no muy detalladamente, la Royal Society tenía noticias de la expedición a Perú a través de la correspondencia mantenida con la Academie des Sciences de París. En mayo de 1745 Réaumur le había informado a Folkes de la conferencia pronunciada en París por La Condamine sobre la expedición y su regreso a través del Amazonas, por lo que eran conocedores de la relevancia científica de la misión.

Gracias a Ulloa, podían tener ahora una información de primerísima mano y un relato completo de las observaciones efectuadas por un científico que había participado directamente en las mismas. De hecho, en las sesiones del 8 y 29 de mayo se leyó un resumen del diario de Ulloa efectuado por el propio Folkes (JBC, 19, p. 101 y pp. 108-120). El documento original, conservado en los Archivos de la Royal Society, consta de 34 páginas y se denomina "An Abstract of Don Antonio de Ulloa's Journal of the observations made by the French Astronomers and himself in Peru./by the President." (Archivo de la RS, Manuscritos: Letters \& Papers, I, 479).

9 Instrucción reservada de lo que de orden del rey debe observar el Capitán de Navío D. Jorge Juan... Museo Naval, ms. 2162, ff. 2-4. Reproducido en LAFUENTE, A.; PESET, J.L. (1981): "Política científica y espionaje industrial en los viajes de Jorge Juan y Antonio de Ulloa (1748-1751)", Melanges de la Casa de Velázquez, 17, 233-262; pp. 249-252. Para la estancia de Jorge Juan en Londres y su 
ficaba que, con el pretexto de "tratar con los individuos de la Regia Sociedad sobre puntos de matemáticas", debía realizar una amplia labor de información, cuya finalidad principal era la de mejorar la construcción naval en nuestro país. Debería procurar para ello la contratación de ingenieros constructores de barcos, además de informarse detalladamente de los métodos ingleses de construcción naval, así como averiguar todos los aspectos que considerase de interés referentes a la política y policía portuaria, y de otros muchos aspectos de carácter político y económico ${ }^{10}$.

Con este motivo, en marzo de 1749 partió Jorge Juan hacia Londres, en donde permaneció algo más de un año. Durante su estancia, además de las actividades propias de su misión ${ }^{11}$, entró en contacto con la Royal Society y fue nombrado miembro de la misma ${ }^{12}$. A su regreso le fue encomendada la Dirección de la Construcción de los buques de la Real Armada, y poco después, en 1752, se estableció el Nuevo Método Español de Construcción Naval, que básicamente combinaba la tradición espa-

misión secreta véase MORALES (1973), "Jorge Juan en Londres", Revista General de Marina, junio 1973, 663-670; y sobre todo el ya citado LAFUENTE-PESET (1981).

10 Textualmente, se decía: "Luego que llegue a Londres echará la voz de que su viaje no tiene otro objeto que el de tratar con los individuos de la regia Sociedad sobre puntos de matemáticas, y para que así se crea los visitará D. Jorge de cuando en cuando." (LAFUENTE-PESET (1981), p. 249). Debían, asimismo, informarse y enviar detallados planos para mejorar la fábrica de nuestros navíos. Igualmente, debían dar información acerca de temas de neto carácter político y económico, como el cumplimiento del tratado de Utrecht, la política proteccionista inglesa, el comercio con la América española y las posibilidades de exportación de productos españoles a Inglaterra.

La misión, que sin duda hoy la calificaríamos como de espionaje militar e industrial, distaba de ser un fenómeno insólito en la época, como en el mencionado trabajo de Lafuente-Peset se pone de relieve al mencionar diferentes misiones encomendadas a otros oficiales como Dámaso Latre y Joaquín Hurtado, o Francisco de Estachería y Joseph Manes (LAFUENTE-PESET (1981), Nota 2, p. 244). Además, no sólo en nuestro país se realizaban este tipo de misiones, pues como indica $\mathrm{M}$. Bradley en un trabajo en el que analiza un caso de espionaje militar e industrial entre Francia y Gran Bretaña, "In fact, the eighteenthcentury was a time of large-scale industrial espionage on the part of all interested western countries". Y más adelante añade: "There can be no doubt that vital military information was crossing the Channel from England, along with many industrial secrets which were transferred to France during the eighteenth century." (BRADLEY, M. (1992): "Engineers as military spies? French engineers come to Britain, 17801790", Ann. Sci., 49, 137-161; pp. 149-150).

11 Durante su permanencia en Londres, obtuvo planos de puertos y arsenales, planos y modelos de barcos de la armada británica, mapas y cartas marinas, datos sobre la actividad en el puerto de Londres, etc. Finalmente, tras diligencias muy laboriosas, y adoptando todo género de precauciones para que no transcendiese su misión, también consiguió "enviar a España tres constructores, un maestro de jarcia, un maestro de lona, operarios y familias" (MORALES (1973), p. 669).

12 El 6 de abril de 1749, fue propuesto como candidato para la Royal Society, siendo su candidatura avalada por Lord Stanhope, M. Folkes, C. Stanhope, Benjamin Robins, John Ellicott y William Watson. La candidatura de Jorge Juan fue votada favorablemente en la sesión del 9 de noviembre de 1749, y fue firmada el 16 del mismo mes. Todos estos detalles se han obtenido del $J B C, 20$, pp. 88-89; p. 162 y pp. 165-66, respectivamente. 
ñola con la técnica naval inglesa ${ }^{13}$. Sin embargo, un mayor afrancesamiento de la corte, junto a la acusación de que tenía "el corazón inglés y odio mortal a todo lo que hace relacion a Francia", hizo que más tarde prevaleciese el sistema francés de construcción, con preferencia al establecido por Jorge Juan ${ }^{14}$.

Los avatares bélicos también motivaron una larga estancia del astrónomo José Joaquín Ferrer en Inglaterra ${ }^{15}$. Apresado en 1780 por la armada inglesa el navío de la Real Compañía Guipuzcoana en el que viajaba a Caracas, fue enviado a Inglaterra. Una vez allí, su familia consiguió que fuese internado en un colegio, en el que permaneció seis años y en donde adquirió una sólida formación en matemáticas y astronomía y un perfecto dominio del inglés. Sus actividades comerciales posteriores le alejaron de Europa, pero siguió manteniendo contacto con Inglaterra, sobre todo con los fabricantes de instrumentos científicos a los que solía recurrir para proveerse de telescopios y otros elementos de observación ${ }^{16}$.

José Rodríguez González, que había tomado parte junto a Biot y Arago en los trabajos de medida del arco de meridiano realizados en nuestro país, fue enviado por el Gobierno español a Inglaterra en 1811, con el objeto de examinar los centros ingleses dedicados a la práctica y enseñanza de la astronomía y sus aplicaciones a la Geografía y a la Navegación ${ }^{17}$. Durante su estancia en Inglaterra escribió un trabajo

13 LAFUENTE, A.; PESET, J.L. (1988): "Las actividades e instituciones científicas en la España Ilustrada", en M. Sellés; J. L. Peset y A. Lafuente (comp.): Carlos III y la ciencia de la Ilustración, Madrid, 29-79; p. 55.

14 Carta de Jorge Juan a Carlos III, reproducida en O'DOGHERTY, P. (1973): “Jorge Juan y la ciencia naval española en el siglo XVIII", Revista General de Marina, junio 1973, 671-689; pp. 678-679.

15 La principal fuente de datos biográficos sobre Ferrer se encuentra en AlCALÁ Galiano, A. (1858): Biografia del astrónomo español don José Joaquín de Ferrer v Cafranga, Madrid. También puede verse J. M. LÓPEZ PIÑERO et al. (1983): Diccionario Histórico de la Ciencia Moderna en España (DHCME), Barcelona, 2 vols.

16 Al menos así lo indica Alcalá Galiano cuando nos dice que a fines de 1813 estuvo en Londres con Mr. Troughton, de quien era corresponsal y amigo íntimo y de quien obtuvo "un magnifico cuarto de circulo, un telescopio de pasajes, dos escelentes barometros de montaña con sus tripodes, un teodolito y varios instrumentos de fisica" (ALCALÁ GALIANO, A. (1858), p. 20).

17 Datos biográficos sobre José Rodríguez se encuentran en DHCME, así como en: FERNÁNDEZ ALONSO, B. (1912): "Discurso en contestación al que para su recepción como académico tenía dispuesto el finado D. Juan Jacobo Durán Loriga”, Boletín de la Real Academia Gallega, Año VII, n ${ }^{\circ}$ 77, 218-227; RODRÍGUEZ CARRACIDO, J. (1917): "La cristalografía en España", en Estudios histórico-críticos de la ciencia española, reimpresión, Barcelona, 1988, pp. 265-272; ALLER, R. M. (1929): “D. José Rodríguez González (O matematico de Bernés), Arquivos Seminario Estudios Galegos, 3, 15-95; ECHARRI, A. (1976): Desarrollo de las matemáticas y la física en España durante el siglo XVIII y algunas causas de la decadencia en el siglo XIX: correcciones a algunos tópicos totalitarios". Comunicación presentada a la 12 Reunión Anual de Matemáticos españoles. Universidad de Málaga, 21-24 de abril; MORENO CASTILLO, R. (1992). Pensamiento matemático en Galicia, Coruña. 
que fue presentado por Mendoza ante la Royal Society en junio de $1812^{18}$, y que apareció publicado poco después en los Philosophical Transactions ${ }^{19}$. En el trabajo se criticaban unas medidas del grado de meridiano efectuadas años atrás por William Mudge, director del Trigonometrical Survey of England ${ }^{20}$. El trabajo de Rodríguez dió origen a dos artículos fuertemente polémicos por parte de Olinthus Gregory ${ }^{21}$, profesor de la Real Academia Militar de Woolwich, que entendió el artículo del español como un ataque al grupo de los "matemáticos prácticos", auspiciado por la dirección de la Royal Society, es decir, por Joseph Banks, cuya amistad con Mendoza y Ríos era bien conocida ${ }^{22}$.

También José Espinosa y Tello permaneció una larga temporada en la capital inglesa $^{23}$. Espinosa fue encargado por el Gobierno español de la recopilación e impresión en Londres de las cartas marítimas más necesarias para la navegación española. Al mismo tiempo se le solicitaban informes y noticias sobre muy diversos aspectos, que iban desde la situación de la marina, el comercio y la pesca en Inglaterra, hasta la descripción de la maquinaria utilizada en sus arsenales o de las casas de moneda. Como resultado de las informaciones allí obtenidas escribió un trabajo sobre la situación de la marina inglesa, publicado años después de su muerte ${ }^{24}$. Una vez concluida la guerra, fue requerido por el Gobierno para que ocupase su anteriores cargos, de los cuales dimitió alegando su mal estado de salud, conservando sólo la Dirección de Hidrografía hasta su fallecimiento ${ }^{25}$.

$18 \mathrm{El}$ artículo fue presentado por Mendoza en las sesiones de la RS correspondientes a los días 4 y 11 de junio de $1812, J B C, 40$, pp. 529-532.

19 RODRÍGUEZ, J. (1812): "Observations on the measurements of three degrees of the meridian conducted in England by Lieut. Col. William Mudge", Philosophical Transactions, 102, 321-351.

20 MUDGE, W (1803): "An account of the measurement of an arc of the meridian, extending from Dunnose, in the Isle of Wight, latitude $50^{\circ} 37^{\prime} 8^{\prime \prime}$, to Clifton in Yorkshire, latitude $53^{\circ} 27^{\prime} 31^{\prime \prime}$, in course of the operations carried on for the Trigonometrical Survey of England, in the years 1800, 1801 and 1802", Philosophical Transactions, 93, 383-508.

21 GREGORY, O. (1813): "Remarks on Don Joseph Rodriguez's animadversions on part of the Trigonometrical Survey of England". Philosophical Magazine, 41, 178-194; GREGORY, O. (1814): "Vindication of the attack on Don Joseph Rodriguez's paper in the Philosophical Transactions", Annals of Philosophy, 3, 282-284.

22 Datos sobre la polémica suscitada por el artículo de Rodríguez se ofrecen en MILLER, D.P. (1983) "Between hostile camps: Sir Humphry Davy's presidency of the Royal Society of London, 1820-1827", British Journal for the History of Science, 16, 1-47; la información sobre la polémica de Rodríguez aparece en las páginas 11-12.

23 Datos biográficos y bibliográficos sobre Espinosa pueden obtenerse, respectivamente, en DHCME y en FERNÁNDEZ DE NAVARRETE, M. (1851): Biblioteca Marítima Española, Vol II, pp. 60-66.

24 Idea de la marina inglesa. escrita por el teniente general de la armada nacional Don José de Espinosa Tello. Mandada imprimir y publicar por las Cortes. Impresa en Madrid, en la Imprenta nacional, año de 1821 . Un cuaderno de 67 páginas en $4^{\circ}$.

$25 \mathrm{Al}$ parecer no se apresuró demasiado para regresar a España, al menos éso es lo que deja traslucir Mendoza en una carta dirigida a Charles Blagden, con fecha de 5 de junio de 1815. En ella se refiere a su 
Pero, sin duda, el científico español que en esta época tuvo una mayor vinculación con Inglaterra fue Don José de Mendoza y Ríos, cuya primera estancia en el Reino Unido en 1780 también fue como prisionero de guerra ${ }^{26}$. Más adelante fue enviado por el Gobierno para viajar por Europa, con el objeto de examinar los avances de otras naciones en navegación y adquirir libros y mapas para formar una biblioteca de marina. Entre 1789 y 1792 residió en París, en donde estableció una duradera relación con alguno de los más eminentes astrónomos de la época y fue nombrado corresponsal de la Academia de Ciencias. A mediados de 1792 se trasladó a Inglaterra, de donde ya no regresaría. Durante la década de los noventa Mendoza fue una especie de embajador científico español en Londres: se encargaba tanto de la tutela de los jóvenes pensionados por el gobierno español en aquella ciudad, como del envío de libros, instrumentos y material científico a España. Asimismo se le encomendó realizase las gestiones para que William Herschel construyera el gigantesco telescopio de 25 pulgadas (el segundo de mayor tamaño de los construidos por el gran astrónomo) para el incipiente Observatorio Astronómico de Madrid ${ }^{27}$. Instado a volver a España por el gobierno, en 1800 fue desposeído de su rango y de todas sus prerrogativas como oficial de la Armada por negarse a regresar a nuestro país ${ }^{28}$.

amigo Espinosa diciendo que tiene "the misfortune of having been appointed to a high place at Madrid, where he must go as the Spanish Government has not accepted his resignation", pero, continua Mendoza, "he has already put off his departure week after week and even month after month" (Archivo RS; General Catalogue Card, BLA.m.15).

26 Tras ser asaltado por piratas ingleses el navío en el que viajaba rumbo a Manila en agosto de 1779, Mendoza permaneció más de un año en la ciudad irlandesa de Cork, hasta que pudo ser canjeado y regresó a Cádiz.

El estudio biográfico más completo y documentado sobre Mendoza se encuentra en ALCALÁ GALIANO, P. (1875): "Estudio sobre la vida y las obras del célebre marino Don José de Mendoza y Ríos", Revista de España, 42, 28-54]. Aspectos parciales de su actividad pueden verse en SELLES, M. (1987a), "La astronomía náutica en la España ilustrada. El tratado de Mendoza y Ríos", Asclepio, 39, 3347; LAFUENTE, A.; Selles, M. A. (1985): "The problem of longitude at sea in the 18th century in Spain", Vistas in Astronomy, 28, 243-250; MERAS, L. M. (1990): "Proyectos cartográficos de la marina ilustrada”, En J. Fernández, I.; González Tascón (eds.): Ciencia. Técnica y Estado en la España Ilustra$d a$, Zaragoza, 367-380, especialmente pp. 373-380.

27 Información acerca de este telescopio construido por Herschel para el Observatorio de Madrid se puede encontrar en CARRASCO, P. (1931): "El gran telescopio de Herschel-Mendoza, adquirido para Madrid a fines del siglo XVII", Revista de la Real Academia de Ciencias exactas Físicas y Naturales de Madrid, 26, 51-59; TiNOCO, J. (1951): Apuntes para la historia del Observatorio de Madrid, Madrid; BENNET, J. (1976): “On the power of penetrating into space: the telescope of William Herschel”, J. Hist. Astronomy, 7, 75108; véanse especialmente pp. 93-95; y, sobre todo, en LÓPEZ ARROYO, M; GIMÉNEZ CAÑETE, A. (1979): "El telescopio Herschel de 25 pies del Real Observatorio Astronómico de Madrid", Las Ciencias, 44, 185-199, en el que se da cuenta detallada de las gestiones de Mendoza.

28 No está claro cuál fue el exacto motivo de su expulsión. Probablemente fue su negativa a volver a España, pero hay que tener en consideración que ya desde 1795 se había solicitado su retorno, y había 
Durante el tiempo que vivió en Inglaterra, Mendoza formó parte del cosmopolita grupo de amigos del todopoderoso presidente de la Royal Society Joseph Banks, con el que tuvo una estrecha relación. En 1793 fue nombrado miembro de la Royal Society, de cuya Junta Directiva formó parte en 1803, y dos de sus trabajos aparecieron en los Philosophical Transactions. Gracias a su amistad con Banks consiguió financiación para la edición de sus famosas tablas por parte del Board of Longitude, la East Indian Company, el Almirantazgo y la Trinity House, e incluso obtuvo una recompensa del citado Board of Longitude por la utilidad de sus tablas para el cálculo de la longitud ${ }^{29}$.

\section{Instrumentación científica y Tecnología}

Durante la primera mitad del siglo XVIII, Londres se convirtió en el principal centro de construcción de instrumental científico de precisión, especialmente en el ámbito de la astronomía y la navegación. En general, la preeminencia de Londres en la construcción de instrumentos se asentó en la reputación de algunos grandes establecimientos como los de George Adams, Benjamin Martin, John Dollond o Jesse Ramsdem ${ }^{30}$. No es extraño por ello que hubiese también en este campo un buen número de españoles que viajasen a Gran Bretaña para adquirir instrumentos o para aprender diversas técnicas instrumentales ${ }^{31}$.

recibido severísimas críticas por parte de Varela por la inutilidad, según este último, de su misión. Véase MERAS (1990), pp. 377-378.

29 Buena prueba de la amistad de Mendoza con Banks puede deducirse de la dedicatoria que Mendoza le dedicó en sus tablas de 1805 donde puede leerse, refiriéndose a Joseph Banks: "The veneration been so successfully devoted to the cultivation and promotion of science, and the gratitude I feel for the regard with which you honour me, would alone incline me to seize every opportunity of publicly testifying to you those sentiments."

Aunque quizá el mejor exponente de estas buenas relaciones sea el retrato de Banks, regalado por Mendoza a la RS, y que todavía hoy se puede contemplar en la Sociedad.

30 BRYDEN, D.J. (1992): "Evidence from advertising for mathematical instrument making in London, 1556-1714", Ann. Sci., 49, 301-336, esp. 334-335.

Sobre la importancia de Inglaterra en el comercio de instrumentos científicos existe una abundantísima literatura. Sin ánimo de ser exhaustivos, indiquemos por ejemplo los artículos de BENNET, J. A. (1985): "Instrument makers and the "decline of science in England": the effect of institutional change on the elite makers of the early nineteenth century", en P. R. de Clerq (ed.): Nineteenth-centurv scientific instruments and their makers, pp. 13-27; COCHRANE, R. C. (1956): "Francis Bacon and the rise of the mechanical arts in Eighteenth-century England", Annals of Science, 12, 137-156; HACKMANN, W. D. (1979): "The relationship between concept and instrument design in Eighteenth-century experimental science", Annals of Science, 36, 205-224; ROBINSON, E. (1956-1957): "The Lunar Society and the improvement of scientific instruments", Annals of Science, 12, 296-304 y 13, 1-8; TURNER, G. L. E. (1976): "The London trade in scientific instrument making in the 18-th century", Vistas in Astronomia, 20, 173-182.

31 La principal fuente de información para todas las actividades relacionadas con la instrumentación científica en la Armada en el siglo XVIII es FERNÁNDEZ DURO, C. (1876-81), Disquisiciones Náuticas, 6 
Entre ellos cabe señalar el caso de Antonio Fernández Solano, catedrático de Física Experimental de los Reales Estudios de San Isidro de Madrid, que en 1783 viajó a París y Londres para visitar los principales establecimientos y talleres científicos de estas ciudades. Como resultado del viaje, el Gabinete de Física de los Reales Estudios se enriqueció "con los más principales instrumentos de óptica", entre los que merecen destacarse un gran telescopio catadióptrico de Gregori, con pie de movimientos mecánicos sobre limbos graduados y micrométricos, y otro telescopio astronómico de menores dimensiones, construidos ambos por James Short, dos microscopios de J. Cuff, que "se consideraban en Europa como los mas escogidos y excelentes", otro solar para gran cámara oscura, y diversos juegos de prismas y espejos ${ }^{32}$.

Podemos incluir también en este apartado el viaje realizado por Aréjula entre 1789 y 1791 por Inglaterra y Escocia, en el que hizo una recopilación de instrumental para el laboratorio de Química que pensaba establecerse en Cádiz, al mismo tiempo que visitaba diversos centros industriales y mineros, con el objeto exclusivo de obtener información de carácter industrial ${ }^{33}$.

El inicio de las expediciones hidrográficas de la armada en 1783, marcó el comienzo de un período de intensa relación hispano-británica en el terreno de la instrumentación científica. La nueva empresa hidrográfica precisaba de todo un conjunto de instrumentos que sólo Inglaterra podía producir con la calidad requerida. Se activó, además, la política del envío de pensionados a Londres para formarse con los

vols., Madrid (especialmente Vol. 4, capítulos dedicados respectivamente a "Instrumentos náuticos" y "Cronometría").

Para una visión de conjunto más actualizada, pueden consultarse los trabajos de SELLES, M. A. (1990): "La política de instrumentos científicos en la Marina de la Ilustración", en J. Fernández; I. González Tascón (eds.): Ciencia. Técnica y Estado en la España ilustrada, Madrid, 3-12; y SELLES, M. A. (1991a): "El problema de la construcción de instrumentos náuticos en la España del Setecientos", en M. Valera y C. López (eds.): Actas del V Congreso de la Sociedad Española de Historia de las Ciencias y de las Técnicas, 3 vols., Murcia, Vol. III, 1913-1927.

Un interesante enfoque sobre la provisión de instrumentos científicos ingleses en la América colonial se ofrece en GLICK, T. F. (1989): "Imperio y dependencia científica en el XVIII español e inglés: la provisión de los instrumentos científicos", en J. L. Peset (coord.): Ciencia, vida y espacio en Iberoamérica, 3 vols., Madrid, pp. 49-63.

32 Sobre el viaje de Fernández Solano y sus adquisiciones para el Instituto, véase SANTISTEBAN, M. (1875): Breve Historia de los Gabinetes de Física y Química del Instituto de San Isidro de Madrid. Madrid, pp. 14-15. Señalemos que el viaje fue costeado por el propio Fernández Solano, quien además corrió con los gastos del nombramiento de un sustituto durante el período que permaneció ausente de España.

33 CARrillo, J. L.; GaGo, R. (1975): "Un aspecto de la comunicación científica entre España y Europa en los siglos XVIII y XIX: Juan Manuel de Aréjula (1755-1830)". Cuadernos de Historia de la Medicina Española, 14, 209-226, esp. pp. 212-214. Véase también la reseña biográfica de Aréjula aparecida en GAGO, R.; CARRILLO, J.L. (1979): La introducción de la nueva nomenclatura química $v$ el rechazo de la teoría de la acidez de Lavoisier en España. Edición facsímil de las Reflexiones sobre la nueva nomenclatura química (Madrid, 1788) de Juan Manuel de Aréjula. Málaga, pp. 19-23. 
mejores artífices, con la esperanza de poder crear posteriormente en España establecimientos donde reparar e incluso construir nuevos instrumentos ${ }^{34}$. Así, en 1785, Baleato, un operario del Arsenal de Ferrol, fue enviado a Londres para que aprendiese la técnica de dividir limbos graduados -algo que había conseguido Ramsden en Inglaterra unos pocos años antes. Durante su estancia, que se prolongó dos años, estudió la máquina de dividir de Ramsden y construyó otra "que mejoraba a la de aquel célebre artista". A su vuelta, se le puso al frente de un taller de construcción de instrumentos náuticos, en el que construyó un círculo de reflexión y después varios instrumentos más: un octante, un sextante, etc.. En 1799 montó el faro de la torre de Hércules, en la Coruña, que se había construido en Londres bajo la dirección de Mendoza ${ }^{35}$.

En 1788 también estuvo pensionado en Londres Amaro Fernández, que construyó "dos agujas náuticas de nueva invención, una máquina eléctrica y otros instrumentos que se remitieron a Madrid"36. A su regreso, en 1793, se establecería un taller-escuela en la Isla de León, que se proyectaba como parte del nuevo Observatorio de Marina ${ }^{37}$.

En 1793, fue el tinerfeño Miguel Borges quien partió hacia Londres "para perfeccionarse en el arte en la casa y fábrica del maestro Stancliffe" 38 . También a principios de los noventa fueron enviados a Londres para formarse en la construcción de instrumentos, Mario Fernández y Carlos Rodríguez, para que a su regreso se hicieran cargo del taller de instrumentación dependiente de la escuela de astronomía de Madrid ${ }^{39}$.

El descubrimiento del cronómetro y su utilización para el cálculo de la longitud en el mar, hizo nacer una incipiente industria de fabricación de los mismos en Francia e Inglaterra. En nuestro país se intentó crear, al menos, un taller de relojería en el que se estuviese en condiciones no sólo de la conservación de los cronómetros, sino de su fabricación. Para ello se enviaron diversos pensionados a París y Londres a trabajar con los mejores constructores de cronómetros, para que a su regreso se pudiesen hacer cargo de la tarea. El primero de los pensionados fue Cayetano Sánchez, alumno destacado de la Escuela de Relojería de Madrid, quien marchó en 1789 a París para trabajar con Berthoud, y en 1792 a Londres junto a Emery. A su regreso se hizo cargo del taller de relojería anejo al Observatorio de Cádiz, en la Isla de León, y en recompensa a su meritoria labor se le concedió el nombramiento de relojero hono-

\footnotetext{
34 SELleS, M. A. (1991b): "Instituciones científicas ilustradas de la Marina", en La ciencia española en Ultramar, Madrid, 97-105, p. 103.

35 FERNÁNDEZ DURO (1876-1881), vol. 4, pp. 72-73.

36 Ibid., p. 73.

37 SELLES (1990), pp. 8-9.

38 El barco en el que regresaba a Canarias en 1799 naufragó, salvándose con once hombres más en un bote que alcanzó la costa africana al sur de Mogador, donde fue apresado, y pudo ser liberado gracias a los esfuerzos del Cónsul de España (FERNÁNDEZ DURO (1876-81), vol. 4, p. 74).

39 Selles, M. A. (1987b): “Astronomía y navegación”, en M. A. Sellés et al (eds.): Carlos III y la ciencia de la Ilustración, Madrid, 81-98; véase p. 87.
} 
rario de la Real Cámara ${ }^{40}$. A su retorno de París y Londres, donde había estado pensionado durante nueve años, Carlos La Rue también se incorporó al taller de relojería, al igual que Eugenio Cruzado. Sin embargo, las fundadas esperanzas que se habían depositado en este taller se vieron desvanecidas con la epidemia de fiebre amarilla que asoló la Isla de León en 1800, y que, entre sus muchas víctimas, se cobró las de Cayetano Sánchez y sus dos ayudantes ${ }^{41}$.

A fines de 1791 se enviaría a Londres a otro de los alumnos destacados de la Escuela de Relojería de Madrid, Antonio Molina, para perfeccionarse junto a Emery en el trabajo de las piedras preciosas aplicadas a los cronómetros. Tras su regreso a España montó el faro de Cádiz en 1795, y fue comisionado para instalar el de la Torre de Hércules en La Coruña. Pero "como si la desgracia señalara a cuantos intentaban en España el adelanto de la relojería", en 1798 falleció repentinamente en París, en donde se encontraba comisionado para la compra de piedras preciosas para el taller del Observatorio ${ }^{42}$.

Señalemos finalmente, en el ámbito de la tecnología, la fructífera estancia en Londres y otras ciudades británicas del más eminente ingeniero español de la época, Agustín de Betancourt, que fue a Inglaterra comisionado por el Gobierno español en dos ocasiones. Durante su primera estancia (1788), que tan solo duró tres semanas, entró en contacto con algunos técnicos relacionados con sus actividades, y estudió el funcionamiento de las máquinas de vapor, que acababan de ser incorporadas a la industria y se convertirían en el principal exponente técnico de la revolución industrial ${ }^{43}$. Durante los tres años que duró su segunda estancia (1793 a 1796) consiguió diversos premios por sus trabajos: uno de ellos otorgado por la Society for the Encouragement of Arts, Manufactures and Commerce ${ }^{44}$, y dos más concedidos por la Royal Society of Agriculture, que además lo nombró socio de mérito. Estudió también la aplicación de la máquina de vapor a los molinos azucareros, inició sus traba-

\footnotetext{
40 FERNÁNDEZ DURO (1876-81), vol. 4, pp. 153-158.

41 Ibid., pp. 158-161.

42 Ibid., pp. 159-160.

43 ROMEU DE ARMAS, A. (1980): Ciencia y tecnología en la España Ilustrada, Madrid; sobre la estancia de Betancourt en Londres, véanse especialmente pp. 176-193.

44 Entre los premios ofrecidos por la Society of Arts en 1794, dentro del apartado de Premiums for invention in Mechanicks el número 227 correspondía a una "Machine for clearing rivers". El premio sería "For the best Model of a Machine, superior to any now in use, for clearing Navigable Rivers from Weeds at the least expence", y debía presentarse en la Sociedad, antes del primer martes de febrero del año 1795. (Transactions of the Society instituted at London for the Encouragement of Arts. Manufactures and Commerce. with the premiums offered in the year 1794, vol. XII, London, 1794). El premio, de 40 guineas, fue obtenido por "Le Chavalier De Betancourt Molina", publicándose su trabajo al año siguiente en la revista de la Society of Arts: "Description of the plate of the machine for cutting weeds in navigable canals and rivers". Transactions of The Society..., vol. XIV (1796), pp. 316-323.
} 
jos sobre telegrafía óptica y adquirió una gran cantidad de material científico con vistas a su frustrada expedición a Cuba.

\section{Botánica}

También se produjo un acercamiento entre los científicos españoles y británicos en el ámbito de la Botánica. Los primeros contactos se debieron principalmente a las relaciones establecidas por Casimiro Gómez Ortega ${ }^{45}$ durante su estancia en 1776 en el Reino Unido, donde visitó los jardines botánicos de Chelsea, Kew y Oxford. Fruto del viaje fue su duradera relación con diversos botánicos ingleses, así como su posterior nombramiento como miembro de la Royal Society y de la Linnean Society.

Otros botánicos españoles pensionados en el Reino Unido fueron los hermanos Claudio y Esteban Boutelou, que recorrieron diversos lugares de Francia e Inglaterra entre 1790 y 1798 para estudiar botánica, agricultura y horticultura. Fruto de su estancia en Inglaterra fue la publicación de sus observaciones sobre la vegetación de los prados artificiales y naturales ingleses según el sistema linneano ${ }^{46}$.

Domingo Badía y Simón Rojas Clemente permanecieron también una temporada en Londres preparando un viaje al mundo islámico; realizaron allí algunos trabajos botánicos, y mantuvieron relación con Joseph Banks, Herschel, Smith, etc. ${ }^{47}$.

Aunque no estuvo en Inglaterra, no podemos dejar de mencionar entre los botánicos españoles relacionados con colegas del Reino Unido al abate Cavanilles, pues

45 Para la estancia de Gómez Ortega en Inglaterra y sus conexiones posteriores, véase PUERTO SARmiento, F. J. (1992): Ciencia de cámara. Casimiro Gómez Ortega (1741-1818) el científico cortesano, Madrid, pp. 75-77 y sobre todo PUERTO SARMIENTO, F. J. (1988): La ilusión quebrada. Botánica. sanidad y política científica en la España Ilustrada, Barcelona, especialmente pp. 152-160 y 180-186, en donde se ofrece una amplia información sobre la estancia y las relaciones de Gómez Ortega con botánicos ingleses.

46 DHCME.

47 Durante su estancia en la capital inglesa se entrevistaron, entre otros, con William Herschel, según consta en una nota de Badía conservada en los archivos del eminente astrónomo. La nota dice así:

"Badia, et Clemente, Envoyés par le Roy d'Espagne faire un Voyage scientifique en Afrique, demandent a Mr Herschell la permission de passer lui rendre ses respects. A cet grace sera particulierment reconnaissant. Badia". (Royal Astronomical Society, Manuscripts Herschel, B. 167).

También se entrevistaron con Edward Smith, presidente de la Sociedad Linneana, según manifiesta Cavanilles en una carta dirigida a Smith: "Je viens d'apprendre que mon eleve cheri Clemente vous a fait une visite. Quel Bonheur pur lui!" (Archivos de la Linnean Society, Smith MS, vol. 3. n 111).

Por otra parte, González Bueno, que se refiere a Simón Rojas Clemente como "un autodidacta, prototipo de erudito ilustrado", nos informa también que "por razones políticas (la participación en una misión de espionaje), habría de formarse en 1802 junto a los más destacados botánicos ingleses: J. Sowerby, J.E. Smith y D. Turner entre otros" (GONZÁLEZ BUENO, A. (1990): "Penetración y difusión de las teorías botánicas en la España ilustrada", en J. Fernández; I. González Tascón (eds.): Ciencia. Técnica $v$ Estado en la España Ilustrada, Zaragoza, 381-395, p. 391). 
fue miembro de la prestigiosa Linnean Society desde 1792 y mantuvo un interesante contacto epistolar con el fundador y primer presidente de la misma, Edward Smith ${ }^{48}$. También Carlos Gimbernat, pensionado para ampliar estudios en Inglaterra en los años noventa ${ }^{49}$, fue miembro de la Linnean Society, al igual que Antonio Palau, José Pavón ${ }^{50}$, y Mariano Lagasca.

Este último, durante su largo exilio londinense, estuvo en relación con varios eminentes botánicos como Robert Brown, John Lindley o Georges Bentham, aunque su contacto más estrecho fue con William Hooker. Gracias a ellos obtuvo facilidades para trabajar en el Jardín Botánico de Chelsea, así como su nombramiento como miembro de la Sociedad Linneana, eximiéndole del pago de cuotas y derechos de entrada. Le facilitaron así mismo la publicación de algunos trabajos referidos a diversos jardines españoles en The Gardener's Magazine ${ }^{51}$.

\section{Medicina}

En el ámbito de la medicina hubo también diversos médicos españoles pensionados en la segunda mitad del XVIII al Reino Unido, entre los que cabe señalar a Antonio de Gimbernat y a José María Ruiz de Luzuriaga. Ambos trabajaron en los Hospitales Saint Thomas y Guy's de Londres y asistieron a las enseñanzas de John Hunter ${ }^{52}$. Ambos estuvieron también una temporada en Edimburgo, en cuya Universidad Ruiz de Luzuriaga se doctoró en Medicina y fue discípulo, entre otros, de Joseph Black y de William Cullen; además, fue nombrado miembro de las Sociedades de Medicina y de Historia Natural de esta ciudad ${ }^{53}$.

48 La correspondencia se encuentra en los Archivos de la Linnean Society y consta de 29 cartas escritas entre 1789 y 1804: Archivos de la LS, Smith MSS, vol 3, números comprendidos entre 64 y 114.

49 Para la biografía de C. Gimbernat debe consultarse SOLÉ, L. (1982): La vida atzarosa del geòleg barceloni Carles de Gimbernat (1768-1834), Barcelona; así como SolÉ, L. (1982): "Diario inédito del geólogo catalán Carlos de Gimbernat (1768-1834)”, Llull, 5, 111-131.

50 Tras la muerte de Hipólito Ruiz, Pavón intentó infructuosamente continuar con la publicación de la Flora peruviana et chilensis. Con el fin de obtener fondos para ello, vendió ejemplares a Philip Barker Webb y a Aylmer Bourke Lambert, traductor al inglés de varias disertaciones botánicas de Ruiz, y por mediación del cual fue elegido miembro correspondiente de la Sociedad Linneana en 1820 (DHCME).

51 LLORENS (1968), p. 45. En esta misma obra se ofrece un resumen de los tres artículos publicados en The Gardener's Magazine, pp. 349-351.

52 Una amplia biografía de Gimbernat aparece en el extenso estudio biobibliográfico del Dr. Salcedo, que constituye el primer volumen de GIMBERnAT, A. (1926-27): Obras de Don Antonio de Gimbernat precedidas de un estudio biobibliográfico del mismo, escrito por el Dr. Enrique Salcedo y Ginestal, 2 vols., Madrid. Sobre la estancia de Gimbernat en Londres y Edimburgo véanse pp. 185-198 del primer volumen.

53 Para la estancia de Luzuriaga en el reino Unido, pueden consultarse: USANDIZAGA, M. (1964): Los Ruiz de Luzuriaga. eminentes médicos vascos "ilustrados", Salamanca; RIERA, J. (1975): "Los estudios en el Reino Unido de Ignacio María Ruiz de Luzuriaga, documentos y epistolario, 1785-1787", 
Entre los profesionales de la medicina exiliados en 1823 se encontraban Juan Manuel de Aréjula, Mateo Seoane y Pablo Montesino. Aréjula, químico y médico, una de las máximas figuras científicas de la España ilustrada, alcanzó renombre internacional con una obra acerca de la fiebre amarilla, y con sus trabajos sobre la nueva nomenclatura química. Debido a su avanzada edad, no cabe reseñar actividad científica alguna en esta su segunda estancia en Inglaterra ${ }^{54}$. Montesino fue el responsable de las primeras escuelas de párvulos y la nueva organización de la enseñanza elemental en nuestro país a partir de 1835, fruto en gran medida de sus observaciones y estudios durante su exilio en Inglaterra ${ }^{55}$.

Pero la figura científica más destacada del exilio español de 1823 fue Mateo Seoane y Sobral ${ }^{56}$, uno de los primeros organizadores de la sanidad militar y pública en España, y junto con Ruiz de Luzuriaga, una de las figuras clave de la medicina social española. Ambos estuvieron profundamente influidos por el mundo ideológico y científico británico, y conformaron una orientación de las disciplinas médicosociales españolas según los modelos británicos. Luzuriaga, como higienista, estuvo directamente influido por el sanitary movement británico de fines del setecientos, mientras que Seoane fue seguidor de la siguiente etapa de la higiene pública inglesa, la dominada ideológicamente por el utilitarismo de Jeremy Bentham ${ }^{57}$.

Entre las muchas actividades de Seoane durante su exilio londinense, cabe destacar su colaboración en el periódico Athenaeum, a cuya redacción perteneció, y en cuyas páginas escribió muchos artículos sobre literatura española, y probablemente casi todos los referidos a sus compañeros de emigración ${ }^{58}$. Fue también, al igual que Lagasca, profesor del Ateneo Español de Londres, institución dedicada a la enseñanza gratuita de los hijos de los emigrados ${ }^{59}$.

Cuadernos de Historia de la Medicina Española, 14, 269-301; GAGO, R.; CARRILlo, J.L. (1981): "La obra fisiológica de Ignacio María Ruiz de Luzuriaga (1763-1822) y su plagio del científico británico Adair Crawford (1748-1795)", Dynamis, 1, 87-100.

54 GARCÍA BALLESTER, L.; CARRILLO, J. L. (1974): “The repression of medical science in absolutist Spain: the case of Juan Manuel de Aréjula 1755-1830". Clio Medica, 9 (3), 207-211. Véase también el ya citado GAGO-CARRILLO (1979).

55 LLORENS (1968), p. 33. Montesino sólo permaneció dos años en Inglaterra, pues pasó después a la isla de Jersey, cerca de Francia. Para una exposición de sus ideas pedagógicas, puede consultarse SAMA, J. (1888): Montesino y sus doctrinas pedagógicas, Barcelona.

56 Sobre Seoane véase LOPEZ PIÑERO, J. M. (1984): Mateo Seoane. La introducción en España del sistema sanitario liberal (1791-1870), Madrid.

57 LÓPEZ PIÑERO, J. M. (1976): Medicina moderna y sociedad española. Siglos XVI-XIX, Valencia. Para Seoane y Luzuriaga, véanse pp. 263-365.

58 LLORENS (1968), p. 348.

59 LLORENS (1968), pp. 76-77. 


\section{RELACIONES INSTITUCIONALES: FELLOWS ESPAÑOLES EN LA ROYAL SOCIETY}

El siglo XVIII fue el siglo de las instituciones científicas. Junto a las creadas en el siglo anterior, cuya máxima expresión fueron la Royal Society de Londres y la Académie des Sciences de París, surgieron por toda Europa, y aun en otros continentes, instituciones científicas con el ánimo de promover el conocimiento en su correspondiente área geográfica de influencia. Estas instituciones rápidamente establecían lazos con entidades análogas o con científicos particulares de otros países, creándose así una vasta red de conexiones internacionales, sobre todo en la segunda mitad del siglo XVIII.

Nuestro país no fue ajeno a esta "fiebre" de instituciones, y aparecieron así la Regia Sociedad de Sevilla de Medicina y Otras Ciencias que, aunque fundada a principios de siglo, adquirió carta de naturaleza realmente en la década de los años treinta, época en la que también se fundó la Academia de Medicina Matritense. Más tarde, ya en la segunda mitad del Setecientos, se fundaría la Academia de Ciencias y Artes de Barcelona, pero quedaría como intento fallido la creación de la Academia de Ciencias de Madrid, para la que fue diseñado el palacio que albergaría posteriormente el Museo del Prado. De este modo Austria y España fueron los dos únicos países europeos de cierto fuste, que carecieron de una Academia central de Ciencias, ubicada en su capital y gozando de fuerte protección oficial, lo que en una época de fuerte centralismo indica una carencia que se dejará sentir en el ámbito institucional de la ciencia española.

La pertenencia a sociedades o instituciones científicas británicas de la época es, por tanto, otro aspecto que nos proporciona interesante información sobre las relaciones de los científicos españoles con el Reino Unido. Sin ánimo de ser exhaustivos, hemos indagado sobre estas relaciones centrándonos en la principal de la época, la Royal Society, pues las restantes asociaciones científicas creadas en el siglo XVIII en Inglaterra carecieron de su importancia o tuvieron un ámbito de influencia fue mucho más reducido. Además, salvo en el caso de la Linnean Society, a la que nos hemos referido al comentar las relaciones en el campo de la botánica, en el curso de nuestra investigación no hemos detectado prácticamente relación alguna con otras instituciones.

Desde la fundación de la Royal Society en el siglo XVII y a lo largo del primer tercio del siglo XVIII, las relaciones entre España y la ilustre Sociedad londinense fueron prácticamente inexistentes, pues hay que esperar a 1716 para encontrarnos con el primer fellow español. En el siglo XIX sucederá casi lo mismo, pues sólo habrá una incorporación en 1819 (y no se producirá ninguna más hasta la de Cajal a principios de siglo). Sin embargo a lo largo del siglo XVIII, en especial en las décadas centrales del siglo, el número de asociados españoles a la Sociedad experimentó un considerable aumento, aunque siempre manteniéndose en cifras muy lejanas a las correspondientes a otros países de nuestro entorno, como Francia o Italia ${ }^{60}$.

60 Véase, por ejemplo, HALL, M. B. (1982-83): “The Royal Society and Italy 1667-1795”, Notes 
En la siguiente relación se indican los nombres de los miembros españoles ${ }^{61}$ de la Royal Society en los siglos XVIII y XIX, junto con las fechas de sus respectivos nombramientos:

5-abril-1716: Marqués de Monte Leone

16-noviembre-1732: Conde de Montijo

13-junio-1734: Diego de Revilla

9-diciembre-1736: Joseph Cervi

11-diciembre-1746: Antonio de Ulloa

9-noviembre-1749: Jorge Juan

9-noviembre-1749: Duque de Medina-Sidonia

6-junio-1751: Marsilius Venturi

8-marzo-1753: Joseph Ortega

31-mayo-1753: Richard Wall

7-diciembre-1758: Joseph Ignacio de Torres

24-enero-1760: Jacobo Rodríguez Pereira

11-junio-1761: Martín Panzano

11-enero-1770: Príncipe de Masserano

6-junio-1776: Pedro Franco Dávila

5-junio-1777: Casimiro Gómez Ortega

20-enero-1780: Bernardo de Belluga

8-marzo-1792: Rev. Thomas Hussey

11-abril-1793: Joseph Mendoza y Ríos

1-abril-1819: Felipe Bauzá

De estos 20 asociados a la Royal Society, cuatro eran embajadores españoles ante el Reino Unido en el momento de su nombramiento: Monte Leone, Montijo, Wall y Masserano; tres más realizaban tareas diplomáticas para la embajada española: Panzano, Belluga y Hussey; y otro más, el duque de Medina Sidonia, fue nombrado simplemente por haber sido recomendado por Ulloa. Dos de ellos realizaron toda su actividad fuera de nuestro país: Revilla en Italia y Pereira en Francia. Quedan, por

and records of the Royal Society of London, 37, 63-81, en donde se recogen más de un centenar de fellows italianos durante el período comprendido en el estudio; o véase también, LAMOINE, G. (1993): "L'Europe de l'esprit ou la Royal Society de Londres", Dix-Huitième Siècle, n 25, 167-198, en donde se ofrece una relación de los 571 fellows extranjeros de la Royal Society, desde su creación hasta 1800, con indicación de su nacionalidad.

61 Al hablar de miembros españoles de la Royal Society nos referimos tanto a los nacidos en España (considerada según sus actuales límites) como a los que, aun naciendo en otros países, fueron nombrados fellows por la actividad realizada en nuestro país. 
tanto, sólo diez miembros cuyo nombramiento fue debido a las tareas científicas realizadas en nuestro país. De ellos, tres eran médicos: Cervi, Venturi y Torres; otros tres naturalistas: Hortega, Dávila y Gómez Ortega; y los cuatro restantes marinos científicos: Ulloa, Jorge Juan, Mendoza y Bauzá. De todos ellos los que mantuvieron una relación mas duradera con la Sociedad fueron Ulloa y, sobre todo, Mendoza, como anteriormente indicamos.

El hecho de que casi la mitad de estos fellows no fuesen científicos no debe extrañarnos, pues hasta mediados del siglo XIX la Royal Society no estuvo limitada exclusivamente a hombres de ciencia. De hecho, eran admitidos en la Sociedad personas que no tenían muchos conocimientos científicos, ni siquiera demasiado interés en la ciencia ${ }^{62}$. Al no contar con apoyo económico oficial como otras sociedades, la Royal Society intentó obtener el apoyo de los poderosos, adoptando la política de reclutar parte de sus miembros entre los sectores ricos e influyentes de la sociedad con algún interés por la ciencia, o por el avance del conocimiento científico ${ }^{63}$. Además, en todo momento prestó especial atención a embajadores y príncipes extranjeros como medio para obtener un mayor reconocimiento internacional.

\section{CONCLUSIÓN}

Como hemos visto, durante la segunda mitad del siglo XVIII y el primer tercio del XIX, hubo un relativamente importante número de científicos españoles que, por unos u otros motivos, llevaron a cabo cierta actividad científica en el Reino Unido, donde establecieron relaciones con personas e instituciones británicas, por lo que, desde esta perspectiva, cabe hablar de una fluida relación científica anglo-española durante la época mencionada. Sin embargo, salvo que investigaciones más detalladas nos aporten nueva información, este flujo casi permanente de hombres de ciencia españoles hacia el Reino Unido tuvo en la mayoría de los casos un aspecto meramente puntual e individual, sin que hayamos podido apreciar una relación duradera o estable con científicos e instituciones británicas, salvo en muy contadas ocasiones. Probablemente, las carencias del sistema educativo de nuestro país, la consiguiente debilidad de las instituciones científicas y la práctica inexistencia de una adecuada infraestructura de investigación, fueron las causas principales de que las relaciones

62 LYONS, H.G. (1939): "The composition of the fellowship and the Council of the Society". Notes and Records of the Royal Society of London. 2 (1939): 108-126, p. 108. Precisamente ésta es una de las características importantes de la Royal Society, la de acoger en su seno gentes de letras y de ciencias a la vez, lo que no ocurría en otras sociedades como la Academia de Ciencias francesa, la de San Petersbourg, etc. "La RS ne sépare pas les talents, elle les rassemble." (LAMOINE, G. (1993), p. 167).

63 LYONS (1939), p. 109. 
MANUEL VALERA, JUAN FRANCISCO LÓPEZ SÁNCHEZ Y CARLOS LÓPEZ FERNÁNDEZ

científicas entre España y el Reino Unido no resultasen plenamente fructíferas, debido a su efímero carácter en la mayoría de los casos.

\section{RECONOCIMIENTO}

Queremos agradecer la ayuda facilitada en la consulta de diverso material de archivo al personal de las Bibliotecas de la Linnean Society, de la Royal Astronomical Society, de la Society of Arts y, muy especialmente, de la Royal Society. Igualmente queremos manifestar nuestro agradecimiento al personal de la Biblioteca del Wellcome Institute for the History of Medicine, y muy especialmente a Mr. Price por su inestimable ayuda durante nuestra estancia en el Instituto. 